\title{
Cost-effectiveness analysis reveals microsurgical varicocele repair is superior to percutaneous embolization in the treatment of male infertility
}

\author{
Jason Ronald Kovac, MD, FRCSC; $;^{*}$ Jake Fantus, MD; ' Larry I. Lipshultz, MD; ${ }^{\dagger}$ Marc Anthony Fischer, MD, \\ FRCSC,'s Zachery Klinghoffer, MD, FRCSC ${ }^{*}$
}

‘Urology of Indiana, Carmel, IN; 'Baylor College of Medicine, Houston, TX; BDivision of Urology, McMaster University, Hamilton, ON; *Urologist, Rogue Valley Health System, ON

Cite as: Can Urol Assoc J 2014;8(9-10):e619-25. http://dx.doi.org/10.5489/cuaj.1873 Published online September 9, 2014.

\section{Abstract}

Introduction: Varicoceles are a common cause of male infertility; repair can be accomplished using either surgical or radiological means. We compare the cost-effectiveness of the gold standard, the microsurgical varicocele repair (MV), to the options of a nonmicrosurgical approach (NMV) and percutaneous embolization $(\mathrm{PE})$ to manage varicocele-associated infertility.

Methods: A Markov decision-analysis model was developed to estimate costs and pregnancy rates. Within the model, recurrences following MV and NMV were re-treated with PE and recurrences following PE were treated with repeat PE, MV or NMV. Pregnancy and recurrence rates were based on the literature, while costs were obtained from institutional and government supplied data. Univariate and probabilistic sensitivity-analyses were performed to determine the effects of the various parameters on model outcomes. Results: Primary treatment with MV was the most cost-effective strategy at \$5402 CAD (Canadian)/pregnancy. Primary treatment with NMV was the least costly approach, but it also yielded the fewest pregnancies. Primary treatment with PE was the least cost-effective strategy costing about $\$ 7300 \mathrm{CAD} /$ pregnancy. Probabilistic sensitivity analysis reinforced $M V$ as the most cost-effective strategy at a willingness-to-pay threshold of $>\$ 4100 \mathrm{CAD} /$ pregnancy.

Conclusions: MV yielded the most pregnancies at acceptable levels of incremental costs. As such, it is the preferred primary treatment strategy for varicocele-associated infertility. Treatment with PE was the least cost-effective approach and, as such, is best used only in cases of surgical failure.

\section{Introduction}

Infertility affects about $15 \%$ of couples in the United States, with approximately $50 \%$ due to a male factor. ${ }^{1}$ Consisting of a dilated and tortuous conglomeration of refluxing pampi- niform plexus veins, varicoceles are present in $15 \%$ of the general population, about $35 \%$ of men with primary infertility and $80 \%$ of those with secondary infertility. ${ }^{2-4}$ While varicoceles are the most easily treatable cause of male infertility, these vascular malformations are associated with a progressive worsening of testicular function if left untreated. ${ }^{5-7}$ Varicocele repair improves semen quality and sperm DNA integrity, ${ }^{8}$ increases clinical pregnancy and live birth rates during in-vitro fertilization (IVF) via intra-cytoplasmic sperm injection (ICSI) $)^{9}$ and potentially recovers semen parameters in men with nonobstructive azoospermia. ${ }^{10,11}$ While the effects of varicocele repair are well-established, the method of correction that is most cost-effective, is controversial.

Numerous surgical techniques exist to correct varicoceleinduced infertility. The current gold standard is the open microsurgical varicocele repair (MV) via either an inguinal or sub-inguinal incision. Both approaches allow for the spermatic cord to be delivered into the incision, making it easy to identify the artery, veins and lymphatics. ${ }^{2}$ In a recent review, the microsurgical subinguinal technique was found to yield the highest pregnancy rates, fewest recurrences and lowest complication rates. ${ }^{4}$ Another surgical option, the nonmicrosurgical varicocele repair (NMV), is still currently practiced, but is not considered to be standard of care. ${ }^{2}$

Another, non-surgical treatment option for varicocele repair is percutaneous embolization (PE). Typically performed by an interventional radiologist, $\mathrm{PE}$ is the selective embolization of gonadal veins. Using coils, PE has the suggested advantages of faster recovery time and protection of the testicular artery without the requirement for anesthesia. PE is unfortunately accompanied by higher failure rates (4\%-11\%) and increased rates of recurrences. ${ }^{2}$ Studies have identified similar improvements in semen parameters and pregnancy rates compared to surgical correction. ${ }^{12-14}$ Interestingly, a recent retrospective review of 158 patients post-PE noted very low failure rates for unilateral, left-sided 
Kovac et al.

embolization prompting the authors to suggest that men with unilateral left-sided varicoceles could be offered either MV or a PE with good expectant outcomes. ${ }^{15}$

When considering varicocele repair in the modern era, the rise of assisted reproductive technology (ART) needs to be considered. The widespread expansion of IVF has resulted in patients with a correctible varicocele offered immediate IVF instead of male factor treatment. ${ }^{1}$ While IVF is effective, this ART approach is still very expensive and has implications for both the offspring and mother, including multiple gestations, low birth weights and possibly increased birth defects. ${ }^{1}$ Moreover, in a recent decision analysis by Meng and colleagues, ${ }^{1}$ varicocele repair was more cost effective than ART while, at the same time, providing comparable live birth rates. ${ }^{1}$ Moreover, in cases of varicocele-associated infertility, immediate IVF should rarely be considered as the favoured treatment strategy. ${ }^{16}$ Indeed, the results of these previously cited studies have almost uniformly demonstrated that initial correction of the underlying cause is the more cost-effective strategy. ${ }^{16,17}$

To date, there are very few studies comparing different methods of surgical correction to themselves and/or to PE. There is a further paucity in the literature with respect to decision analysis and cost-effectiveness regarding these different approaches. As such, the purpose of our current study was to comprehensively analyze the cost-effectiveness of various surgical approaches available to correct varicoceleassociated male infertility. Specifically, we employed a decision-analysis model to determine whether MV, MNV or PE would yield the best treatment strategy with respect to costs, complications, fertilization and live birth rates.

\section{Methods}

\section{Model design}

Classification of treatment arms was determined based on currently accepted techniques and a review of recent literature. ${ }^{15,18-20}$ The various strategies include NMV, MV and PE (Table 1). The treatment strategies were organized with decision arms progressing from primary to secondary treatment approaches over time (Fig. 1). Exclusion of the retroperitoneal, scrotal and laparoscopic options was made given the relative infrequency with which these surgeries were performed and the associated lack of comprehensive costing and outcomes. ${ }^{21,22}$

A decision tree was constructed (Fig. 1) using decision analysis software (TreeAge Pro Healthcare 2009, TreeAge Software Inc., Williamstown, MA.). Each treatment strategy

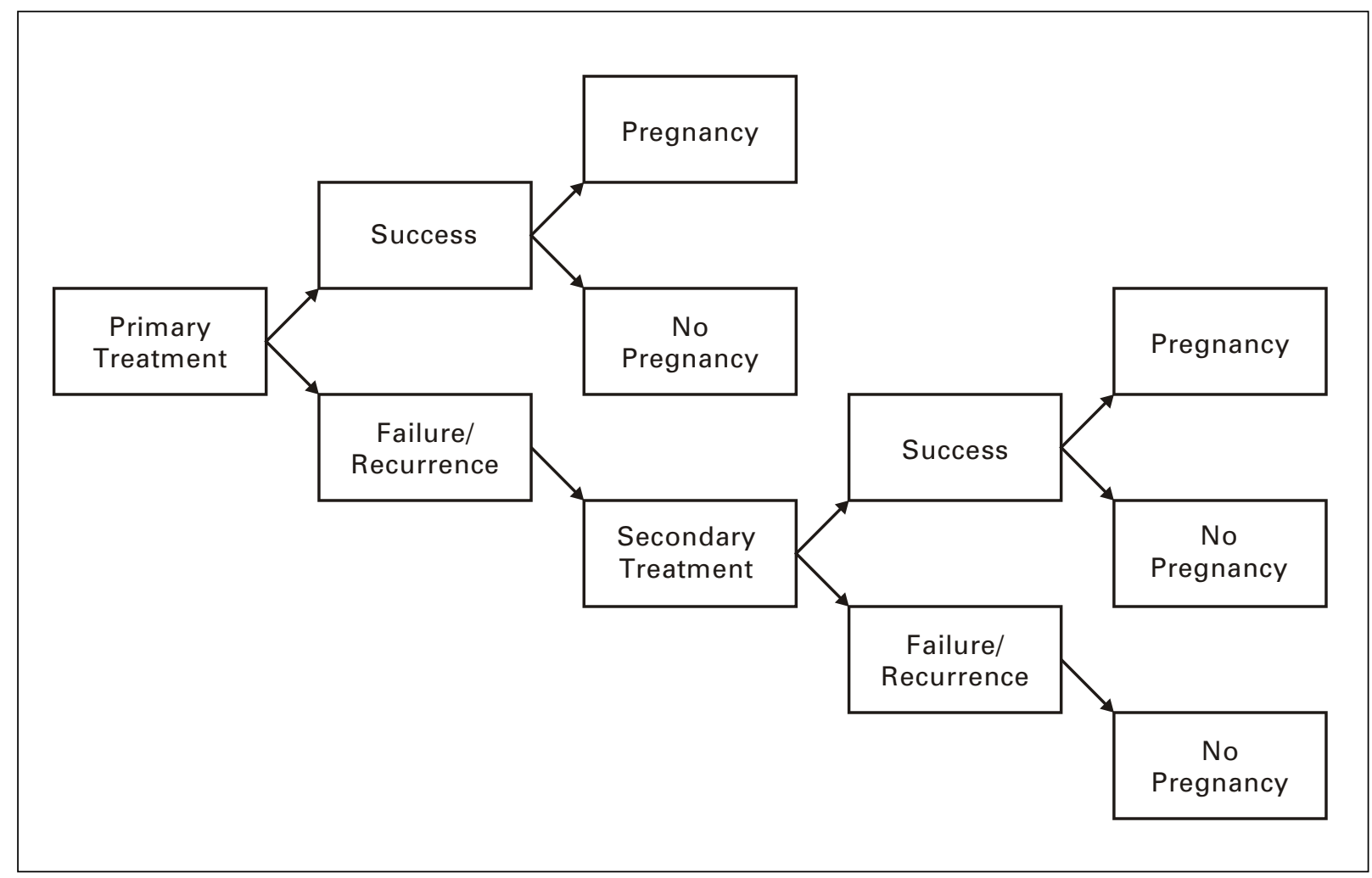

Fig. 1. Decision tree. 


\begin{tabular}{|c|c|c|}
\hline Treatment strategy & Primary treatment & Secondary treatment \\
\hline 1 & NMV & PE \\
\hline 2 & MV & $\mathrm{PE}$ \\
\hline 3 & $\mathrm{PE}$ & NMV \\
\hline 4 & PE & MV \\
\hline 5 & PE & PE \\
\hline
\end{tabular}

consisted of a primary treatment followed by a secondary treatment for recurrences or treatment failures (Table 1, Fig. 1). The decision to exclude surgical approaches (NMV, MV) as secondary treatments for primary treatment surgical failures (Table 1) was made given the lack of literature available for this treatment condition. A Markov simulation cycle was then developed to estimate costs and pregnancy rates, as well as to evaluate each type of procedure (NMV, NMV and PE). Within the model, recurrences following MV and NMV were re-treated with PE and recurrences following PE were treated with repeat PE, MV or NMV (Fig. 1). Primary or secondary treatments complicated by a hydrocele could then proceed to hydrocele repair. Other complications of varicocele repair were excluded from the model. The time to progress from the start of the decision tree to the Markov cycle was considered immediate and no time was accrued during this period. Costs, pregnancy rates and recurrencefree rates were computed for each treatment strategy by performing 10000 Monte Carlo micro-simulations.

\section{Model data sources}

\section{Probability estimates}

Varicocele recurrence rates (Table $2 \mathrm{a}$ ), pregnancy rates (Table 2 b) and hydrocele formation rates (Table 2c) following MV, NMV and PE were obtained from a pooled analysis of 33 studies evaluating varicocele repair outcomes by Diegidio and colleagues. ${ }^{4}$ Reported recurrence rates were assumed to represent both recurrences and treatment failures.

Estimated recurrence rates following NMV, MV and PE as primary treatments were $15.7 \%, 2.1 \%$ and $4.3 \%$, respectively (Table 2a). Recurrence rates following each procedure as a secondary treatment was estimated as $110 \%$ of

\begin{tabular}{lcc}
\hline Table 2b. Pregnancy rates & & \\
\hline Technique & Mean (\%) & Range (\%) \\
\hline NMV & 30.1 & $20.0-31.5$ \\
MV & 44.8 & $33.8-51.5$ \\
PE & 31.9 & $12.2-40.0$ \\
\hline NMV: non-microsurgical approach; PE: percutaneous embolization; MV: microsurgical \\
varicocele repair.
\end{tabular}

\begin{tabular}{lcc}
\hline Table 2a. Recurrence rates & & \\
\hline Technique & Mean (\%) & Range (\%) \\
\hline NMV & 15.7 & $3.6-17.5$ \\
MV & 2.1 & $0.7-15.2$ \\
PE & 4.3 & $3.6-17.5$ \\
\hline
\end{tabular}

NMV: non-microsurgical approach; PE: percutaneous embolization; MV: microsurgical varicocele repair.

their respective primary recurrence rate. This was done to reflect a possible decreased success rate in patients already demonstrating a predilection to recurrence as previously described. ${ }^{23,24}$

Estimated pregnancy rates following NMV, MV and PE were $30.1 \%, 44.8 \%$ and $31.9 \%$, respectively (Table $2 b$ ). In simulations where a secondary treatment was required for recurrence, the pregnancy rate was estimated as the lesser of the primary and secondary procedure types.

Estimated hydrocele formation rates following NMV, MV and PE were $7.5 \%, 0.7 \%$ and $0 \%$, respectively (Table 2c). Reports describing the frequency that patients with postvaricocele repair hydroceles pursued repair was not available; however, we estimated this frequency as $25 \%$ based on expert opinion. All hydrocele repairs were assumed to be successful and the complications of the hydrocele repair were not considered.

\section{Cost estimates}

Costs were estimated from a payer perspective. Detailed cost derivations in Canadian and US (United States) dollars were determined and summarized (Table $3 a$, Table $3 \mathrm{~b}$ ). US dollar costs were based on current (2013) conversion rates. The direct costs of NMV, MV and PE were estimated from a retrospective review of institutional cost data at a Canadian Hospital.

Hospital costs included the use of operating room, operating room personnel, surgical equipment, disposables, use of post-anesthetic recovery room and medications used in hospital (Table 3a, Table 3b). The surgeon, radiologist and anesthesia fees were based on the 2012 Ontario Health Insurance Plan (OHIP) fee schedule (Table 3a, Table 3b). ${ }^{25}$ All procedures were assumed to be outpatient procedures requiring no hospital admission. The capital cost and depreciation of the operative microscope was not considered since it is typically available at most institutions with costs

\begin{tabular}{lcc}
\hline \multicolumn{3}{l}{ Table 2c. Hydrocele rates } \\
\hline Technique & Mean (\%) & Range (\%) \\
\hline NMV & 7.5 & $4.3-17.5$ \\
MV & 0.7 & $0.0-1.6$ \\
PE & 0.0 & 0.0 \\
\hline NMV: non-microsurgical approach; PE: percutaneous embolization; MV: microsurgical \\
varicocele repair.
\end{tabular}


Kovac et al.

\begin{tabular}{lcc}
\hline \multicolumn{3}{l}{ Table 3a. Cost estimates for NMV and MV* } \\
\hline & NMV & MV \\
\hline Hospital costs & $\$ 1175$ (range: $\$ 702-$ & $\$ 1711.13$ (range: \\
& 1619 ) (\$1184 USD) & $\$ 1224.56-3304.56)$ \\
Surgeon fee & $\$ 205.35$ (\$206.87 USD) & $\$ 205.35$ (\$206.87 USD) \\
Anesthesia fee & $\$ 225.15$ (\$226.82 USD) & $\$ 315.21$ (\$317.54 USD) \\
Total cost & $\$ 1605.50(\$ 1617.39$ & $\$ 2231.69$ (\$2248.21 \\
& USD) & USD) \\
\hline *Cost \$CAD (\$US). NMV: non-microsurgical approach; MV: microsurgical varicocele repair.
\end{tabular}

shared among several surgical services.

Indirect patient costs included costs of outpatient analgesia routinely prescribed and health-related productivity loss (HRPL) or societal costs (Table 4a, Table 4b). HRPL was calculated by incorporating the mean time off work (Table 4a) multiplied by the mean Canadian hourly wage for males (Table 4b). The mean time off work and the average wages were obtained from Canadian Government source documents. The costs of the operative hydrocele repair were calculated using similar methodologies.

\section{Univariate and probabilistic sensitivity analysis}

The robustness of our model to variations in key parameters was first analyzed by performing a univariate sensitivity analysis. Each parameter was individually varied across a clinically plausible range of values and the outcome of the model was recalculated throughout this range. Although univariate sensitivity analysis can identify the relative influence of individual parameters on model outcome, it inadequately reproduces real-world variability where multiple parameters may change simultaneously.

We thus addressed these limitations by performing a probabilistic sensitivity analysis, which realistically reflects real world uncertainty by varying each model parameter simultaneously. This analysis was done by substituting each parameter estimate with a probability distribution and by performing a Monte Carlo simulation. In these simulations, we estimated a theoretical patient's progress through the decision analysis model with parameter values randomly drawn from each probability distribution. Thus, probability distributions were created around each parameter using the variance reported in the literature. ${ }^{26}$

\begin{tabular}{lcc}
\hline Table 4a. Societal costs & & \\
\hline Technique & Mean (days) & Range (days) \\
\hline NMV & 6.6 & $3-9$ \\
MV & 4.8 & $1-8$ \\
PE & 1.0 & NA \\
\hline
\end{tabular}

NMV: non-microsurgical approach; PE: percutaneous embolization; MV: microsurgical varicocele repair; NA: not available.

\begin{tabular}{lc}
\hline \multicolumn{2}{l}{ Table 3b. Cost estimates for PE* } \\
\hline Hospital costs & $\$ 1907.94$ (range: \$1840.65-2477.45) \\
& $(\$ 1922.07$ USD) \\
Radiologist fee & $\$ 317.40$ (\$319.75 USD) \\
Anesthesia fee & $\$ 0$ (\$0 USD) \\
Total cost & $\$ 2225.34$ (\$2241.82 USD) \\
\hline${ }^{*}$ Cost $\$$ CAD (\$US). PE: percutaneous embolization.
\end{tabular}

Clinically plausible estimates of variance were used when no published variance data were available. ${ }^{26}$ Following standard conventions, costs were modelled with gamma distributions and transition probabilities were modelled with beta distributions. The results of 1000 Monte Carlo simulations were plotted on a cost-effectiveness axis. A cost-effectiveness acceptability curve (CEAC) was then generated by determining the percentage of simulations that remained cost-effective over a range of willingness-to-pay (WTP) thresholds. Reported WTP thresholds, which reflect the highest additional cost infertile couples are willing to pay for one additional pregnancy, ranged from $\$ 15000$ to $\$ 65000$ USD. 4,16,26 There is currently no consensus on society's WTP threshold for providing an infertile couple with one additional pregnancy.

\section{Results}

The results of the index case cost-utility analysis are summarized in Table 5. PE followed by PE was the least costly strategy at $\$ 2538$ CAD, but had the second lowest pregnancy rate at 0.319 pregnancies. MV followed by PE was the strategy that achieved the highest pregnancy rate (0.444), although it did so at a higher cost (\$3271 CAD). Adjusting the cost per pregnancy, MV-PE was superior at a cost of $\$ 7363$ CAD per pregnancy. The incremental cost-effectiveness ratio (ICER) of MV-PE was \$5569 CAD per pregnancy. Given a WTP range from $\$ 15000$ to $\$ 65000$ CAD, this highlights MV-PE as the preferred strategy with a higher effectiveness versus cost ratio (Fig. 2).

The remaining strategies: PE-NVM, PE-MV and NMV-PE all failed to show any increase in pregnancy rate compared to MV-PE $(0.316,0.319,0.299$, respectively). The higher associated costs of PE-NVM, PE-MV and NMV-PE compared to $\mathrm{PE}-\mathrm{PE}$ resulted in higher costs per pregnancy.

Recurrence, pregnancy and hydrocele rates following NVM, MV, and PE were described above in Table 2a, Table $2 \mathrm{~b}$ and Table 2c. Using a WTP of $\$ 15000$ to $\$ 60000$ $\mathrm{CAD}, \mathrm{MV}-\mathrm{PE}$ was the preferred strategy at all ranges of sam-

\begin{tabular}{lcc}
\hline Table 4b. Lost wages & & \\
\hline $\begin{array}{l}\text { Average wage } \\
\text { (per hour) }\end{array}$ & $\begin{array}{c}\text { Work hours } \\
\text { (per day) }\end{array}$ & Average wage (per day) \\
$\$ 25.58$ CAD (\$25.77 US) & 8 & $\$ 204.64$ CAD (\$206.16 US) \\
\hline
\end{tabular}




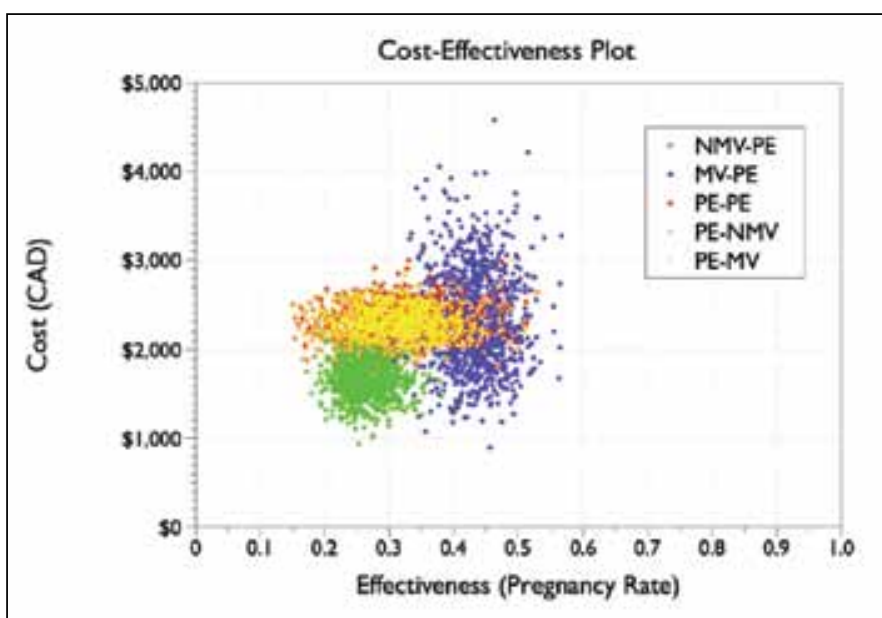

Fig. 2. A cost-effectiveness plot demonstrating the superiority of microsurgical varicocele repair-percutaneous embolization (MV-PE) as the most costeffective treatment approach.

pled parameters. The cost-effectiveness acceptability curve (CEAC) (Fig. 3) shows a change from PE-PE at \$5790 USD to MV-PE. Using a WTP of $\$ 15000$ to $\$ 60000 \mathrm{CAD}$, it can be concluded that MV-PE is the most cost-effective strategy.

\section{Discussion}

Our study demonstrates that in varicocele-associated causes for male infertility, MV resulted in higher pregnancy rates than any of the other treatment strategies. In addition, it did so with an acceptable ICER (\$5569/pregnancy), making it the most cost-effective strategy in our decision-analysis. PE was not as cost-effective and should therefore be saved for varicoceles that have previously failed surgical management. Both strategies are within the WTP threshold of $\$ 15000$ to $\$ 60000$ CAD with MV-PE still preferred. MV-PE is still preferred in part because of the higher success rates and ultimately, the lower achievable costs per pregnancy. Both $P E$ and MV were far superior to NVM given the increased cost per pregnancy and highest complication rates.

To our knowledge, no randomized, controlled clinical studies comparing the outcomes of the different treatment methods for varicocele repair currently exist. In a recently performed prospective, randomized clinical trial, subinguinal MV resulted in improved semen analysis parameters, as well as enhanced rates of spontaneous pregnancies when compared to observation $(13.9 \%$ vs. $32.9 \%)$ with a number needed to treat of 5.27. ${ }^{27} \mathrm{~A}$ meta-analysis performed by Cayan and colleagues in $2009^{28}$ is currently the benchmark for comparison. The authors analyzed 36 studies from 1980 to 2008 and showed that spontaneous pregnancy rates using MV were $41.97 \%$. This was significantly greater than the $33.2 \%$ using $\mathrm{PE}^{28}$ and similar to the results that we have obtained using our model. Moreover, Cayan and colleague ${ }^{28}$ also demonstrated that NMV had a spontaneous pregnancy rate of $36 \%$, a recurrence rate $2.63 \%$, and a hydrocele formation rate of $7.3 \%$. The laparoscopic technique, which was not included in the current study, has a less successful $30.07 \%$ spontaneous pregnancy rate with a recurrence rate of $4.3 \%$. Given the significantly inferior pregnancy rates obtained using a laparoscopic approach, it was excluded from our study. ${ }^{28}$

With regards to cost-effectiveness, several studies have been performed looking at male infertility secondary to the presence of a varicocele. An early study by Schlegal and colleagues in $1997^{17}$ evaluated whether using ART as the primary method of treatment for varicocele-associated male infertility was more cost-effective compared to surgical correction. When male factor infertility was bypassed via direct treatment using ART, men with varicoceles had an average cost per successful delivery of $\$ 89091$ USD. ${ }^{17}$ This was significantly more than the $\$ 26268$ USD cost per delivery for men who had their varicoceles surgically repaired prior to ART. ${ }^{17}$ The authors concluded that specific treatment for varicocele-associated infertility with surgical repair was more cost-effective than primary treatment with ART. More recently, Penson and colleagues ${ }^{16}$ noted similar findings: immediate IVF was not as cost-effective as varicocele repair followed by IVF. Specifically, immediate IVF accrued average costs of \$20 394 USD, with a live birth effectiveness probability of $0.61 .^{16}$ This was more expensive and less effective than initial varicocele repair followed by IVF ( $\$ 15980$ USD, effectiveness probability $=0.72) .{ }^{16}$ Interestingly, when the couples were 'treated' with observation (assumed to be at a cost of \$0), the cost per live pregnancy was, as would be expected, substantially less than varicocele repair followed by IVF. Given that observation alone was effective $14 \%$ of the time, the cost-savings occurred at the expense

Table 5. Index case cost-utility analysis

\begin{tabular}{|c|c|c|c|c|}
\hline Treatment strategy & Cost \$CDN (\$USD) & Pregnancy rate & $\begin{array}{l}\text { Cost per pregnancy, cost } \\
\text { SCDN (SUSD) }\end{array}$ & ICER, cost \$CDN (\$USD) \\
\hline PE-PE & $\$ 2538$ (\$2557 USD) & 0.319 & $\$ 7964$ (\$8023 USD) & - \\
\hline PE-NMV & $\$ 2565$ (\$2584 USD) & 0.316 & $\$ 7964$ (\$8023 USD) & Dominated \\
\hline PE-MV & $\$ 2574$ (\$2593 USD) & 0.319 & $\$ 8068$ (\$8128 USD) & \$122 775/pregnancy (\$123684 USD) \\
\hline MV-PE & $\$ 3271$ (\$3295 US) & 0.444 & $\$ 7363$ (\$7418 USD) & $\$ 5569 /$ pregnancy (\$5610 USD) \\
\hline NMV-PE & $\$ 3412$ (\$3437 USD) & 0.299 & $\$ 11429$ (\$11 514 USD) & Dominated \\
\hline
\end{tabular}




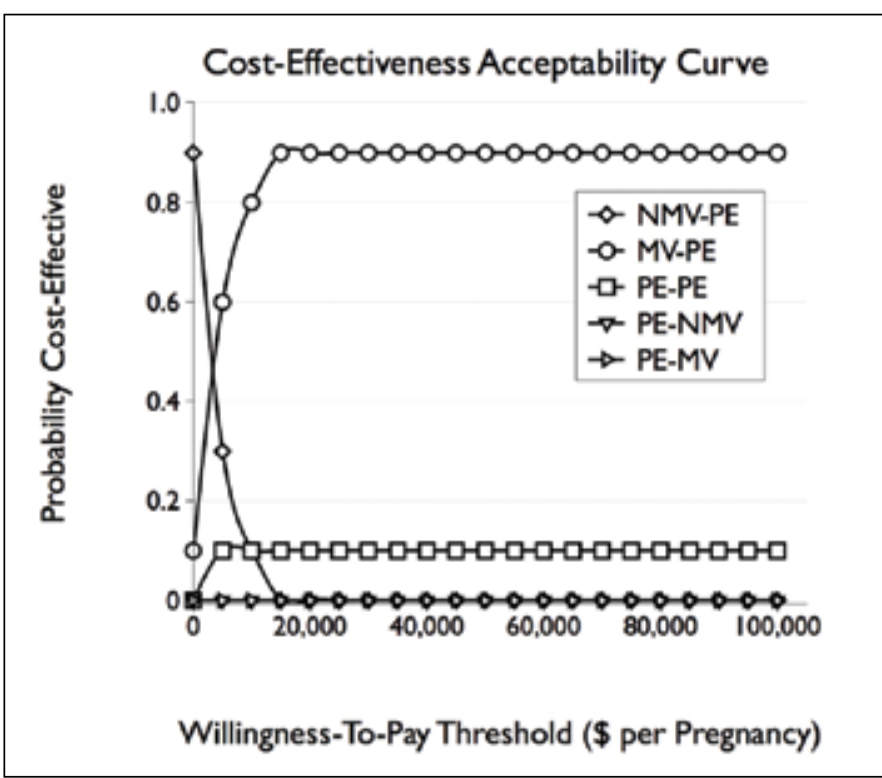

Fig. 3. The cost-effectiveness acceptability curve concluding that microsurgical varicocele repair-percutaneous embolization (MV-PE) is the most cost-effective strategy.

of success. ${ }^{16}$ When multiple pregnancies were considered, varicocele repair followed by IVF dominated immediate IVF without surgical repair. ${ }^{16}$

While we did not consider multiple pregnancies within our decision analysis model, the current study has other limitations. Similar to all models of decision analysis, the quality of data is highly reliant on the information used to populate the model. Since there is a limited amount of literature comparing PE to MV, most of our data came from a single pooled analysis of 33 studies. ${ }^{4}$ Thus, to strengthen our limited data, we used a comprehensive sensitivity analysis to minimize the uncertainty. Ideally, a multicentre randomized control trial comparing the cost of $\mathrm{MV}$ and $\mathrm{PE}$, along with their respective spontaneous pregnancy rates, could be used to resolve the controversy. Further limitations include the fact that the costing was done based on the data from a single Canadian centre. As such, variability would be expected both regionally and internationally.

\section{Conclusion}

In the current study, the cost-effectiveness of surgical varicocele repair was compared to PE. MV yielded the greatest number of pregnancies at an acceptable level of incremental cost. Based on these findings, MV should be the first-line treatment for varicocele-related infertility. Conversely, we found that PE is best reserved to treat varicoceles refractory to surgical management.
Competing interests: Dr. Kovac, Dr. Fantus. Dr. Lipshultz, Dr. Fischer and Dr. Klinghoffer all declare no competing financial or personal interests.

This paper has been peer-reviewed.

\section{References}

1. ReferenceMeng MV, Greene KL, Turek PJ. Surgery or assisted reproduction? A decision analysis of treatment costs in male infertility. J Urol 2005;174:1926-31; discussion 1931.

2. Lipshultz L, Thomas A, Khera M. Surgical Management of Male Infertility. In: Wein A, ed. Campbell-Walsh Urology. Vol. 1. Philadelphia: PA; Saunders; 2007.

3. Dubin L, Amelar RD. Etiologic factors in 1294 consecutive cases of male infertility. Fertil Steril 1971;22:469-74.

4. Diegidio $\mathrm{P}$, Jhaveri JK, Ghannam $\mathrm{S}$, et al. Review of current varicocelectomy techniques and their outcomes. BJU Int 2011;108:1157-72. http://dx.doi.org/10.1111/j.1464-410X.2010.09959.x

5. Cozzolino DJ, Lipshultz LI. Varicocele as a progressive lesion: positive effect of varicocele repair. Hum Reprod Update 2001;7:55-8. htrp://dx.doi.org/10.1093/humupd/7.1.55

6. Pinto KJ, Kroovand RL, Jarow JP. Varicocele related testicular atrophy and its predictive effect upon fertility. J Urol 1994;152:788-90.

7. Witt MA, Lipshultz LI. Varicocele: A progressive or static lesion? Urology 1993;42:541-3. http://dx.doi. org/10.1016/0090-4295(93)90268-F

8. Li F, Yamaguchi K, Okada K, et al. Significant improvement of sperm DNA quality after microsurgical repair of varicocele. Syst Biol Reprod Med 2012;58:274-7. http://dx.doi.org/10.3109/19396368 .2012 .692431

9. Esteves SC, Oliveira FV, Bertolla RP. Clinical outcome of intracytoplasmic sperm injection in infertile men with treated and untreated clinical varicocele. J Urol 2010;184:1442-6. http://dx.doi.org/10.1016/i. juro.2010.06.004

10. Mehta A, Goldstein M. Varicocele repair for nonobstructive azoospermia. Curr Opin Urol 2012;22:507-12. http://dx.doi.org/10.1097/MOU.0b013e328358e27b

11. Kim ED, Leibman BB, Grinblat DM, et al. Varicocele repair improves semen parameters in azoospermic men with spermatogenic failure. J Urol 1999;162:737-40. http://dx.doi.org/10.1097/00005392199909010-00031

12. Dewire DM, Thomas AJ Jr, Falk RM, et al. Clinical outcome and cost comparison of percutaneous embolization and surgical ligation of varicocele. J Androl 1994;15:38S-42S.

13. Nabi $G$, Asterlings $S$, Greene DR, et al. Percutaneous embolization of varicoceles: Outcomes and correlation of semen improvement with pregnancy. Urology 2004;63:359-63. http://dx.doi.org/10.1016/i. urology.2003.09.026

14. Shlansky-Goldberg RD, VanArsdalen KN, Rutter CM, et al. Percutaneous varicocele embolization versus surgical ligation for the treatment of infertility: Changes in seminal parameters and pregnancy outcomes. J Vasc Interv Radiol 1997;8:759-67. http://dx.doi.org/10.1016/S1051-0443(97)70657-2

15. Cassidy D, Jarvi K, Grober E, et al. Varicocele surgery or embolization: Which is better? Can Urol Assoc J 2012;6:266-8.

16. Penson DF, Paltiel AD, Krumholz HM, et al. The cost-effectiveness of treatment for varicocele related infertility. J Urol 2002;168:2490-4. http://dx.doi.org/10.1016/S0022-5347(05)64175-4

17. Schlegel PN. Is assisted reproduction the optimal treatment for varicocele-associated male infertility? A costeffectiveness analysis. Urology 1997;49:83-90. http://dx.doi.org/10.1016/S0090-4295(96)00379-2

18. Kroese AC, de Lange NM, Collins J, et al. Surgery or embolization for varicoceles in subfertile men. Cochrane Database Syst Rev 2012;10:CD000479.

19. Liu $X$, Zhang $H$, Ruan $X$, et al. Macroscopic and microsurgical varicocelectomy: What's the intraoperative difference? World J Urol 2013;31:603-8. http://dx.doi.org/10.1007/s00345-012-0950-x. Epub 2012 Sep 22.

20. Ficarra V, Crestani A, Novara G, et al. Varicocele repair for infertility: What is the evidence? Curr Opin Urol 2012;22:489-94. http://dx.doi.org/10.1097/MOU.0b013e328358e115

21. Chan P. Management options of varicoceles. Indian J Urol 2012;27:65-73. http://dx.doi. org/10.4103/0970-1591.78431

22. Ding H, Tian J, Du W, et al. Open non-microsurgical, laparoscopic or open microsurgical varicocelectomy for male infertility: A meta-analysis of randomized controlled trials. BJU Int 2012;1 10:1536-42. http:// dx.doi.org/10.1111/i.1464-410X.2012.11093.x 
23. Kim J, Shin JH, Yoon HK, et al. Persistent or recurrent varicocoele after failed varicocoelectomy: Outcome in patients treated using percutaneous transcatheter embolization. Clin Radiol 2012;67:359-65. http:// dx.doi.org/10.1016/j.crad.2011.10.007

24. Moon KH, Cho SJ, Kim KS, et al. Recurrent varicoceles: Causes and treatment using angiography and magnification assisted subinguinal varicocelectomy. Yonsei Med J 2012;53:723-8. http://dx.doi. org/10.3349/ymi.2012.53.4.723

25. Care MoHaL-T. Schedule of Benefits for Physician Services under the Health Insurance Act; 2012. http:// www.health.gov.on.ca/english/providers/program/ohip/sob/physserv/physserv_mn.html. Accessed August 4, 2014.

26. Klinghoffer Z, Tarride JE, Novara G, et al. Cost-utility analysis of radical nephrectomy versus partial nephrectomy in the management of small renal masses: Adjusting for the burden of ensuing chronic kidney disease. Can Urol Assoc J 2013;7:108-13.
27. Abdel-Meguid TA, Al-Sayyad A, Tayib A, et al. Does varicocele repair improve male infertility? An evidence-based perspective from a randomized, controlled trial. Eur Urol 2011;59:455-61. http://dx.doi. org/10.1016/j.eururo.2010.12.008

28. Cayan $S$, Shavakhabov $S$, Kadioglu A. Treatment of palpable varicocele in infertile men: A meta-analysis to define the best technique. J Androl 2009;30:33-40. http://dx.doi.org/10.2164/jandrol.108.005967

Correspondence: Urology of Indiana, Male Reproductive Endocrinology and Surgery, 12188-A North Meridian Street, Suite 200, Carmel, Indiana, 46032; ikova@@urologyin.com 